\title{
Das Potenzial genetisch modifizierter und gentechnisch konstruierter Organismen in der wirkungsbezogenen Analytik
}

\author{
Georg Reifferscheid · Sebastian Buchinger · Evelyn Claus
}

Eingegangen: 11. März 2009 / Akzeptiert: 29. April 2009/Online veröffentlicht: 16. Mai 2009

(C) Springer-Verlag 2009

Zusammenfassung Am Beispiel des spezifischen toxikologischen Endpunktes Gentoxizität wird das Potenzial bioanalytischer Instrumente im Rahmen der wirkungsbezogenen Analytik aufgezeigt. Gentoxische Potenziale von Sedimenten verschiedener Flusseinzugsgebiete wie Rhein, Elbe, Donau und Oder wurden mit dem Ames-Fluktuationstest in Kombination mit gaschromatografisch/massenspektrometrischen Analysen untersucht. Mit den Standardteststämmen für den Ames-Fluktuationstest war in Sedimentporenwässern der untersuchten Flüsse nur gelegentlich ein geringes mutagenes Potenzial zu detektieren. Partikelgebundene Gentoxine ließen sich allerdings in mittelpolaren Fraktionen von Sedimentextrakten nachweisen. PAK, von denen mehrere in Annex X der EU-WRRL (Directive 2000/60/ EC) als prioritär gefährlich gelistet sind, tragen hier signifikant zum mutagenen Potenzial bei. Dennoch spielen viele, bisher nicht als prioritär gefährlich definierte Substanzen eine wichtige Rolle. $\mathrm{Zu}$ ihnen zählen z. B. DibenzopyrenIsomere, von denen einige ein erhebliches gentoxisches (und karzinogenes) Potenzial besitzen. Die Einbeziehung metabolisch kompetenter bakterieller Teststämme, welche Enzyme des Fremdstoffmetabolismus exprimieren, erweiterte signifikant das Spektrum nachweisbarer Gentoxine hinsichtlich mittelpolarer bis polarer Verbindungen. Diese Teststämme zeigten auch Mutagenität in Porenwässern frischer Sedimente. Am Beispiel eines Altlastenstandortes im Einzugsgebiet der Elbe wurde eine Reihe von heterozykli-

Verantwortliche Herausgeber: Jan Schwarzbauer · Peter Heininger · Evelyn Claus

G. Reifferscheid $(\bowtie) \cdot$ S. Buchinger $\cdot$ E. Claus

Bundesanstalt für Gewässerkunde,

Referat G3 Ökotoxikologie und Biochemie,

Am Mainzer Tor 1, 56068 Koblenz, Deutschland

E-Mail: reifferscheid@bafg.de schen und N-substituierten Verbindungen nachgewiesen, die das mit metabolisch kompetenten Bakterien detektierbare hohe mutagene Potenzial des Standortes bedingen können.

Schlüsselwörter Ames Fluktuationstest - Effektdirigierte Analyse $\cdot$ Gentechnisch modifizierte Teststämme $•$ Gentoxizität $\cdot$ Mutagenität

The potential of genetically modified and genetically engineered organisms in the effect-directed analysis

Abstract Using the example of the specific toxicological endpoint genotoxicity the potential of bio-analytical instruments as tools in effect-directed analysis is demonstrated. Genotoxic potentials of sediments from different river basins such as the Rhine, Elbe, Danube and Oder were analyzed with the Ames-fluctuation test in combination with gas chromatography/mass spectrometry. In sediment pore waters of the investigated rivers a low mutagenic potential could be measured occasionally when standard test strains were used. However, the fractionation of sediment extracts showed that particle bound genotoxic substances are detectable especially in the medium polar fractions. PAH, including several that are listed as priority hazardous compounds in Annex X of the EU WFD, contribute significantly to the mutagenic potential. Nevertheless, many compounds that were previously not defined as priority hazardous substances play an important role. As an example Dibenzopyrene isomers were identified, some of which have a high genotoxic (and carcinogenic) potential. The extention of the test battery to metabolically competent test strains which express enzymes of xenobiotic metabolism pathways, broadened significantly the range of detectable genotoxins in direction of medium polar to polar compounds. These test strains also 
showed mutagenicity in pore waters of fresh sediments. In a contaminated site in the Elbe catchment area, many heterocyclic and $\mathrm{N}$-substituted compounds were detected, possibly determining the high mutagenic potential of the site.

Keywords Ames Fluctuation test $\cdot$ Effect-directed analysis $\cdot$ Genetically modified test strains $\cdot$ Genotoxicity $\cdot$ Mutagenicity

\section{Problemstellung}

DNA-schädigende (gentoxische) und mutagene (erbgutverändernde) Substanzen in der Umwelt spielen für exponierte Organismen aufgrund potenziell irreversibler Folgen, die auch Prozesse der Karzinogenese begünstigen, eine wichtige Rolle. DNA-schädigende Stoffe kommen natürlicherweise vor, werden aber auch durch anthropogene Aktivitäten in die Umwelt entlassen und reichern sich häufig in hydrophoben Kompartimenten (z. B. Sedimenten) an. Dabei ist zu berücksichtigen, dass die weitaus meisten in der Umwelt vorkommenden Stoffe sogenannte Pre-Mutagene sind. Sie sind per se nicht DNA-reaktiv, sondern werden erst in den Zellen der Organismen durch Stoffwechselreaktionen (z. B. Oxidationsprozesse) intermediär zu bioaktiven Verbindungen umgewandelt. Aus Vorsorgegründen gilt es, Emissionen gentoxischer Verbindungen so weit wie möglich zu verhindern. In den vergangenen Jahren wurden mehrere prokaryontische und eukaryontische Testverfahren für die Untersuchung gentoxischer Wirkungen im aquatischen Bereich entwickelt und standardisiert (z. B. ISO 13829, ISO 16240). Diese Testverfahren zielen in erster Linie auf in der wässrigen Phase gelöste Verbindungen ab. Partikelgebundene Gentoxine lassen sich meist nur nach Extraktion erfassen. Prokaryontische Testverfahren sind zwar robust, schnell und kosteneffizient, die Testorganismen sind aber metabolisch nur eingeschränkt kompetent, was gegenüber organismischen Ansätzen oder solchen mit metabolisch kompetenten Zellkulturen ein Nachteil ist. Aus diesem Grunde wird in neuester Zeit versucht, fremdstoffmetabolisierende Gene des Säugerstoffwechsels in Bakterien zu integrieren und funktionell zu exprimieren (Reifferscheid und Buchinger 2009), um von der auf einen „worst case“ ausgerichteten externen Bioaktivierung mittels metabolisch hoch aktiver Leberhomogenate hin $\mathrm{zu}$ realistischeren Bedingungen mit interner Bioaktivierung zu kommen.

\section{Ziel}

Dieser Beitrag gibt einen Überblick über die mit prokaryontischen in-vitro-Testverfahren erzielten Erfolge in der wirkungsbezogenen Analytik der vergangenen Jahre. Gen- toxizität in Abwässern, Oberflächengewässern und Grundwässern wurde insbesondere mit dem in vitro Comet Assay, dem $и т и$-Test und dem Ames-Test nachgewiesen. Allerdings war es zunächst nur in Einzelfällen gelungen, verantwortliche Substanzen zu identifizieren (Reifferscheid und Grummt 2000; Eisenträger et al. 2007). In der vorliegenden Studie sollte die Identifikation DNA-schädigender Stoffe in aquatischen Kompartimenten durch Einsatz einer Batterie gentechnisch modifizierter Testbakterien mit stufenweise erhöhter metabolischer Kompetenz verbessert werden.

\section{Ergebnisse und Diskussion}

Zur Identifikation sedimentassoziierter Gentoxine wurde der Ames-Fluktuationstest eingesetzt (Reifferscheid et al. 2005a,b; ISO 11350). Dabei handelt es sich um ein bakterielles Testverfahren auf erbgutverändernde Wirkung. Sedimentporenwässer zeigen mit diesem Testverfahren bei Verwendung von Standardteststämmen nach ISO 16240 nur vereinzelt ein DNA-schädigendes Potenzial. Partikelgebundene Gentoxine lassen sich allerdings in mittelpolaren Fraktionen von Sedimentextrakten nachweisen. Polyzyklische aromatische Kohlenwasserstoffe, von denen mehrere in Annex X der EU-Wasserrahmenrichtlinie (Directive 2000/60/EC) als prioritär gefährlich gelistet sind, tragen hier signifikant zum mutagenen Potenzial bei. Benzo(a)pyren $(\mathrm{BaP})$ ist in verschiedenen Fällen eine dominant mutagene Substanz. Dennoch spielen viele, bisher nicht als prioritär gefährlich definierte Substanzen eine wichtige Rolle. Zu ihnen zählen z.B. Dibenzopyren-Isomere, von denen einige ein erheblich höheres gentoxisches (und karzinogenes) Potenzial besitzen als BaP. Im Hinblick auf den Fremdstoffmetabolismus verfügen die Standardteststämme lediglich über die prokaryontische Basisaktivität. Werden durch gentechnische Veränderungen erzeugte metabolisch kompetentere Teststämme eingesetzt, die zwei Enzyme des Fremdstoffmetabolismus exprimieren (Nitroreduktase und Acetyltransferase, Abb. 1), zeigen sowohl Porenwässer als auch fraktionierte Sedimentextrakte erhöhte gentoxische Effekte. Mit Untersuchungen von Sedimentfraktionen lässt sich nachweisen, dass sich das mutagene Potenital vom mittelpolaren bis in den polaren Bereich hinein erstreckt.

Am Beispiel eines belasteten Standortes im Einzugsgebiet der Elbe (Spittelwasserregion) wurden unter Verwendung des Ames-Fluktuationstests mit parallel durchgeführter gaschromatografischer/massenspektrometrischer Analyse polyzyklische aromatische Kohlenwasserstoffe (PAK) sowie heterozyklische und substituierte Stickstoffverbindungen als Hauptverursacher der Mutagenität identifiziert. Letztere sind (Zwischen-)Produkte oder deren natürliche Metabolite aus ehemaliger Farbstoff- und Pflanzenschutzmittelproduktion. Die Mutagenität korrelierte dabei meist mit der zur 
Abb. 1 In dieser Studie verwendete Batterie von Testbakterien mit abgestufter Fremdstoffwechsel-Kompetenz

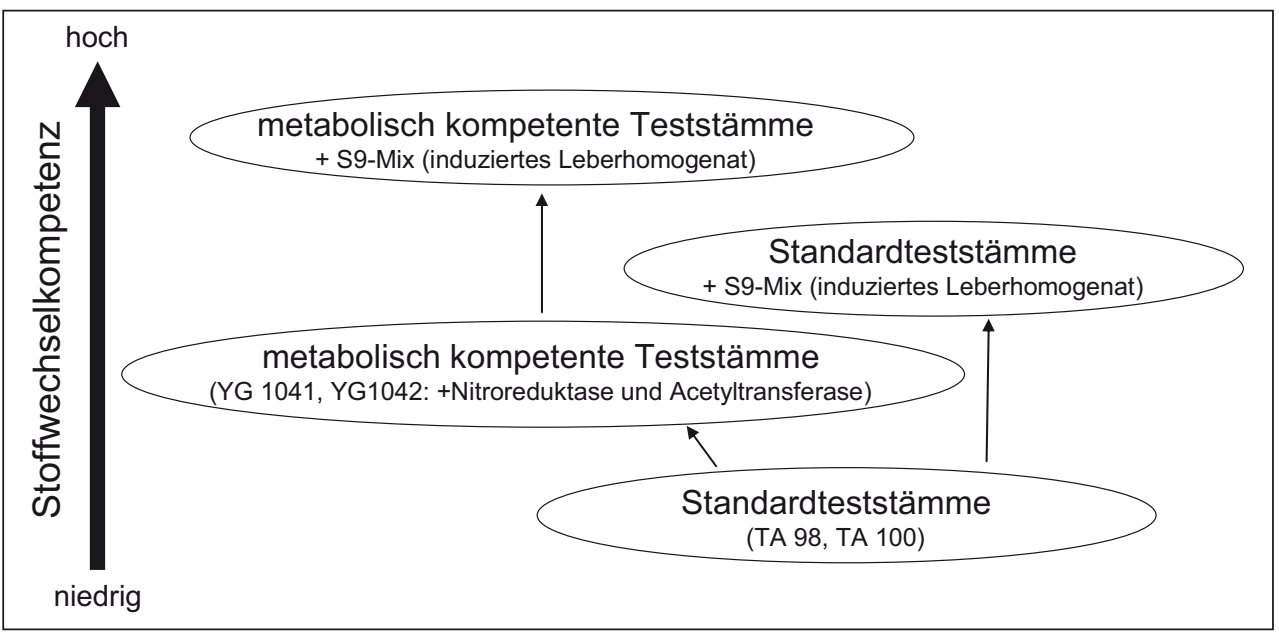

Abb. 2 Mutagenes Potenzial (Rasterschubmutationen) der mittelpolaren Fraktion eines Sedimentextraktes aus der Spittelwasser-Region und Versuch der Identifikation verursachender Substanzen durch wirkungsbezogene Analytik

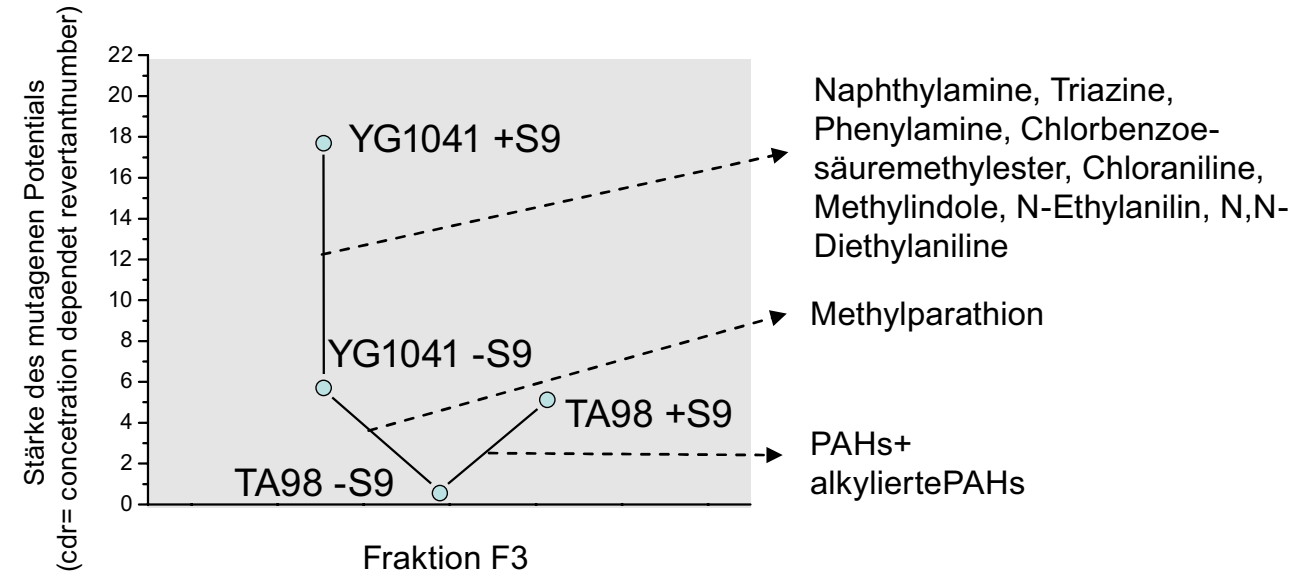

Verfügung stehenden metabolischen Kompetenz (z. B. Standardstamm TA 98 oder TA $100<$ Standardstamm + S9-Mix $\approx$ metabolisch kompetenter Stamm YG1041 oder YG1042 $<$ metabolisch kompetenter Stamm + S9-Mix) (Abb. 2). Es ist möglich, dass Gentoxine aus der Spittelwasserregion, so wie es bereits für Dioxine und Schwermetalle nachgewiesen wurde, bei Hochwasserereignissen remobilisiert und in die Auen der mittleren Elbe eingetragen werden.

\section{Ausblick}

Metabolisch kompetente Bakterienteststämme sollen zukünftig verstärkt zur Aufklärung gentoxischer und mutagener Wirkungen eingesetzt werden. Zudem muss untersucht werden, welches Risiko von den identifizierten Gentoxinen für höhere Organismen ausgeht. Derzeit wird ein internationaler Standard für die Testung auf Umweltmutagenität erarbeitet, der zum ersten Mal metabolisch kompetente bakterielle Teststämme mit einbezieht (ISO CD 11350). In den vergangenen beiden Dekaden etablierte bioanalytische Instrumente für die Messung spezifischer Toxizität, die z. T. auf genetisch modifizierten bzw. gentechnisch veränderten Organismen oder suborganismischen Systemen beruhen, werden heute zwar vielfach eingesetzt, sind aber erst ansatzweise in untergesetzlichen Regularien (Vollzug) implementiert.

Die Instrumente tragen dazu bei, ein Bewusstsein für die Relevanz spezifischer und möglicherweise irreversibel wirkender Schadstoffe in der Umwelt zu schaffen und Maßnahmen zur Reduktion ihrer Emissionen zu ergreifen, damit von der EU-WRRL geforderte Bewirtschaftungsziele für die Flussgebietseinheiten erreichbar werden.

\section{Literatur}

Directive 2000/60/EC of the European Parliament and of the Council of 23 October 2000 establishing a framework for Community action in the field of water policy

Eisenträger A, Reifferscheid G, Dardenne F, Blust R, Schofer A (2007) Characterisation and identification of the hazard of a former ammunition site using microarrays, bioassays and chemical analysis. Environ Toxicol Chem 26(4):634-646

ISO 13829 (2000) Water quality - Determination of the genotoxicity of water and waste water using the $u m u$-test 
ISO 16240 (2005) Water quality - Determination of the genotoxicity of water and waste water - Salmonella/microsome test (Ames test)

ISO CD 11350 (2009) - Water quality - Determination of the genotoxicity of water and waste water - Salmonella/microsome fluctuation test (Ames fluctuation test)

Reifferscheid G, Grummt T (2000) Genotoxicity in German surface waters - results of a collaborative study. Water Air Soil Pollut 123:67-79

Reifferscheid G, Arndt C, Schmid C (2005a) Further development of the $\beta$-lactamase MutaGen assay and evaluation by comparison with Ames fluctuation tests and the $u m u$ test. Environ Molecul Mutagen 46(2):26-139

Reifferscheid G, Claus E, Manz W (2005b) Spezifische toxische Wirkungen in der Sedimentbewertung. Nachweis spezifischer Wirkungen und Identifikation verursachender Stoffe. Wasser Journal 103:3-32

Reifferscheid G, Buchinger S (2009) Cell-based genotoxicity testing. Genetically modified and genetically engineered bacteria in environmental genotoxicology. In: Belkin S, Gu MB (eds) Advances in Biochemical Engineering/Biotechnology. Whole cell sensing systems. Springer, Berlin Heidelberg (im Druck) 